\title{
Mice lacking the signaling molecule CaIDAG-GEFI represent a model for leukocyte adhesion deficiency type III
}

\author{
Wolfgang Bergmeier, ${ }^{1,2}$ Tobias Goerge,1,2 Hong-Wei Wang,1,2 \\ Jill R. Crittenden, ${ }^{3,4}$ Andrew C.W. Baldwin, ${ }^{1}$ Stephen M. Cifuni, ${ }^{1}$ \\ David E. Housman, ${ }^{4}$ Ann M. Graybiel, ${ }^{3}$ and Denisa D. Wagner ${ }^{1,2}$

\begin{abstract}
${ }^{1}$ CBR Institute for Biomedical Research and 2Department of Pathology, Harvard Medical School, Boston, Massachusetts, USA
3Department of Brain and Cognitive Sciences and McGovern Institute for Brain Research and

${ }^{4}$ Center for Cancer Research, Massachusetts Institute of Technology (MIT), Cambridge, Massachusetts, USA.
\end{abstract}

\begin{abstract}
Single gene mutations in $\beta$ integrins can account for functional defects of individual cells of the hematopoietic system. In humans, mutations in $\beta_{2}$ integrin lead to leukocyte adhesion deficiency (LAD) syndrome and mutations in $\beta_{3}$ integrin cause the bleeding disorder Glanzmann thrombasthenia. However, multiple defects in blood cells involving various $\beta$ integrins $\left(\beta_{1}, \beta_{2}\right.$, and $\left.\beta_{3}\right)$ occur simultaneously in patients with the recently described LAD type III (LAD-III). Here we show that the product of a single gene, $\mathrm{Ca}^{2+}$ and diacylglycerol-regulated guanine nucleotide exchange factor I (CalDAG-GEFI), controlled the activation of all 3 integrins in the hematopoietic system. Neutrophils from CalDAG-GEFI ${ }^{-/-}$mice exhibited strong defects in Rap 1 and $\beta_{1}$ and $\beta_{2}$ integrin activation while maintaining normal calcium flux, degranulation, and ROS generation. Neutrophils from CalDAG-GEFIdeficient mice failed to adhere firmly to stimulated venules and to migrate into sites of inflammation. Furthermore, CalDAG-GEFI regulated the activation of $\beta_{1}$ and $\beta_{3}$ integrins in platelets, and CalDAG-GEFI deficiency caused complete inhibition of arterial thrombus formation in mice. Thus, mice engineered to lack CalDAG-GEFI have a combination of defects in leukocyte and platelet functions similar to that of LAD-III patients.
\end{abstract}

\section{Introduction}

Integrins are heterodimeric cell-surface receptors that mediate adhesion to the extracellular matrix and cell-cell interactions. On circulating blood cells, most integrins are expressed in a resting, low-affinity state. Cellular stimulation induces a high-affinity state in the integrins, which enables them to bind to their ligands. The molecular basis of this inside-out activation of integrins is only partially understood (1). In blood cells, integrins are critical for the formation of the immunological synapse (2), in the extravasation of circulating immune cells from the bloodstream (3), and in the formation of platelet plugs at sites of vascular damage (4). The functional importance of integrins expressed on blood cells is best documented in patients with germline mutations in the gene encoding $\beta_{2}$ integrins (leukocyte adhesion deficiency type I; LAD-I; ref. 5), who experience recurrent infections, and in patients with mutations in the genes encoding $\alpha_{\text {IIb }}$ or $\beta_{3}$ integrins (Glanzmann thrombasthenia; ref. 6), who have bleeding diathesis. Similar phenotypes have been observed in mice with specific deletions in the genes coding the $\beta_{2}(7)$ or $\beta_{3}(8)$ integrins.

Recently, patients with normal expression but defective activation of $\beta_{1}, \beta_{2}$, and $\beta_{3}$ integrins have been identified (9-13). Affected patients exhibit clinical symptoms such as severe recurrent infections, a heightened tendency to bleed, and marked leukocytosis. It has been proposed that this group of integrin activation disorders

Nonstandard abbreviations used: CalDAG-GEFI, $\mathrm{Ca}^{2+}$ and diacylglycerol-regulated guanine nucleotide exchange factor I; fMLP, formyl-methionylleucylphenylalanine; GEF, guanine nucleotide exchange factor; LAD, leukocyte adhesion deficiency; $\mathrm{LTB}_{4}$ leukotriene $\mathrm{B}_{4}$; PAF, platelet activating factor; PAR4p, PAR4-activating peptide; PSGL-1, P-selectin glycoprotein ligand-1; TG, thioglycollate.

Conflict of interest: The authors have declared that no conflict of interest exists. Citation for this article: J. Clin. Invest. 117:1699-1707 (2007). doi:10.1172/JCI30575. be designated LAD-III (9) based on the nomenclature for LAD-I and LAD-II, which describes patients with impaired expression of $\beta_{2}$ integrins (5) and defective fucosylation of selectin ligands (14), respectively. Because the expression of $\beta_{1}, \beta_{2}$, and $\beta_{3}$ integrins appears to be normal in LAD-III patients, it seems likely that a genetic defect in 1 or more intracellular signaling molecules involved specifically in the activation of leukocyte and platelet integrins is the basis of the LAD-III syndrome.

We have recently identified $\mathrm{Ca}^{2+}$ and diacylglycerol-regulated guanine nucleotide exchange factor I (CalDAG-GEFI; also referred to as RasGRP2) as crucial for $\beta_{3}$ integrin activation in platelets (15). CalDAG-GEFI is a member of the CalDAG-GEF/RasGRP family of intracellular signaling molecules, containing binding sites for calcium and diacylglycerol as well as a guanine nucleotide exchange factor (GEF) domain that catalyzes the exchange of GTP for GDP bound to Rap1 or Rap2 $(16,17)$. Rap1 is the major isozyme in both platelets and neutrophils $(18,19)$. Interestingly, in contrast to Rap1, which is ubiquitously expressed in hematopoietic and nonhematopoietic cells, CalDAG-GEFI appears to be specifically expressed in platelets and megakaryocytes as well as neutrophils within the hematopoietic system as well as in neurons, especially in the striatum of the basal ganglia $(15,17)$. CalDAG-GEFI ${ }^{-1-}$ mice are characterized by severely impaired hemostasis, caused by defective activation of Rap 1 and integrin $\alpha_{\mathrm{IIb}} \beta_{3}$ in platelets, and by mild neutrophilia (15). This neutrophilia indicated to us that neutrophils from CalDAG-GEFI-/- mice might have a defect in extravasation similar to that observed in selectin-deficient mice (20), causing neutrophil accumulation in the bloodstream. Therefore, we evaluated the role of CalDAG-GEFI in the activation of leukocyte integrins, specifically members of the $\beta_{2}$ integrin family. Our results pointed to significant defects in $\beta_{1}$ integrin-and $\beta_{2}$ integrin-mediated 
Table 1

Expression of adhesion receptors on peripheral blood neutrophils

$\begin{array}{lcccccc} & \text { IgG } & \text { CD18 } & \text { LFA-1 } & \beta_{1} \text { Integrin } & \text { PSGL-1 } & \text { L-selectin }^{A} \\ \text { WT } & 9.5 \pm 1.7 & 456 \pm 81 & 62 \pm 10.7 & 54.2 \pm 8.3 & 1839 \pm 135 & 804 \pm 46.4 \\ \text { CalDAG-GEFF-- } & 10.9 \pm 1.7 & 392 \pm 61 & 62 \pm 9.9 & 35 \pm 2.7 & 1662 \pm 246 & 647 \pm 18.9 \\ P & & 0.5 & 0.9 & 0.03 & 0.6 & 0.01\end{array}$

Peripheral blood neutrophils were stained with fluorophore-labeled antibodies against the indicated adhesion receptors and analyzed by flow cytometry. Results are mean fluorescence intensity \pm SEM, $n=7$. ATwo populations of neutrophils were detected in CalDAG-GEFl-1- mice: one with mean fluorescence similar to that observed in WT neutrophils, the other with mean fluorescence less than $50 \%$ that of WT. A similar decrease in L-selectin was previously observed in our lab in circulating neutrophils from P/E-selectin-deficient mice, which also show a defect in neutrophil extravasation (20). LFA-1, lymphocyte function-associated antigen 1.

lets (15). To determine whether CalDAG-GEFI plays a role in integrin activation in neutrophils, we studied neutrophil responses to activation both in vitro and in vivo in CalDAG-GEFI ${ }^{-1-}$ mice and their littermate WT controls.

We first compared the surface expression of key adhesion receptors in neutrophils from CalDAG-GEFI ${ }^{-/}$and WT mice. As shown in Table 1, CalDAG-GEFI-deficient and WT neutrophils expressed comparable levels of P-selectin glycoprotein ligand-1 (PSGL-1) and $\beta_{2}$ integrins on their surface. No difference in agonist-induced upregulation of $\alpha_{M} \beta_{2}$ (Mac-1) on the cell surface was observed, demonstrating that knockout neutrophils were fully capable of recruiting

adhesion of CalDAG-GEFI-deficient neutrophils in vitro and in vivo, which caused a markedly impaired response to acute inflammation. Furthermore, we examined the function of CalDAG-GEFI in platelets and found that CalDAG-GEFI was essential for the activation of $\beta_{1}$ integrins on platelets and that arterial thrombus formation was completely abolished in CalDAG-GEFI-/- mice. Thus CalDAG-GEFI regulates $\beta_{1}, \beta_{2}$, and $\beta_{3}$ integrins, which suggests this gene may be defective in patients with LAD-III.

\section{Results}

Normal expression of agonist receptors, calcium flux, and ROS formation in CalDAG-GEFI-deficient neutrophils. Integrin activation plays a key role in the firm adhesion and extravasation of PBLs (3). We have previously shown that CalDAG-GEFI is expressed in platelets and neutrophils and that it is critical for $\beta_{3}$ integrin activation in plate- intracellular pools of integrins by granule release (Figure 1A). Compared with WT neutrophils, CalDAG-GEFI-deficient neutrophils expressed less L-selectin and $\beta_{1}$ integrin on their cell surface.

To study signaling responses triggered by surface-expressed agonist receptors, we tested several agonists for their ability to induce calcium flux in neutrophils from WT and CalDAG-GEFI-/- mice. No differences were observed in calcium flux in response to various doses of $\mathrm{C} 5 \mathrm{a}$ or leukotriene $\mathrm{B}_{4}\left(\mathrm{LTB}_{4}\right.$; Figure $\left.1 \mathrm{~B}\right)$ or formylmethionylleucylphenylalanine (fMLP; data not shown) between WT and mutant cells, nor did we detect significant differences in the formation of ROS between WT and CalDAG-GEFI-deficient neutrophils stimulated with AMLP or PMA (Figure 1C). This finding is in line with previous studies showing that the small GTPase Rac (not a target of CalDAG-GEFI) is required for the activation of the respiratory burst in neutrophils $(21,22)$. These data establish
A
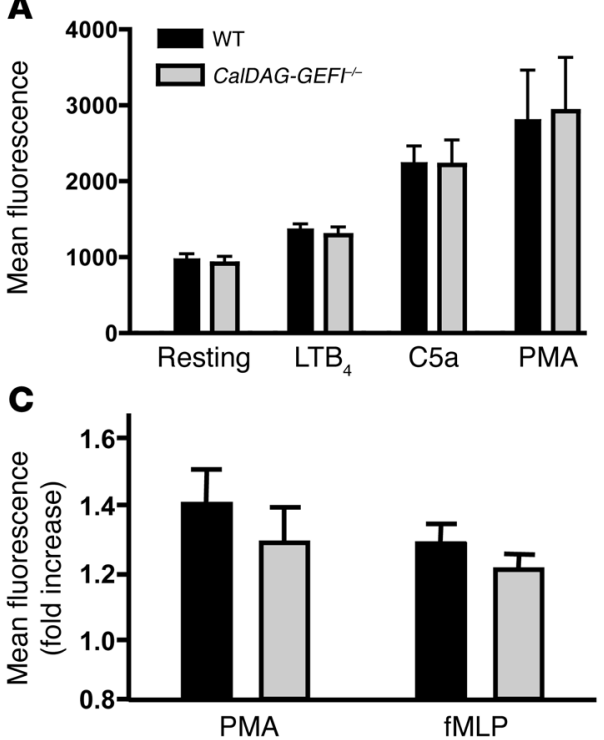

B
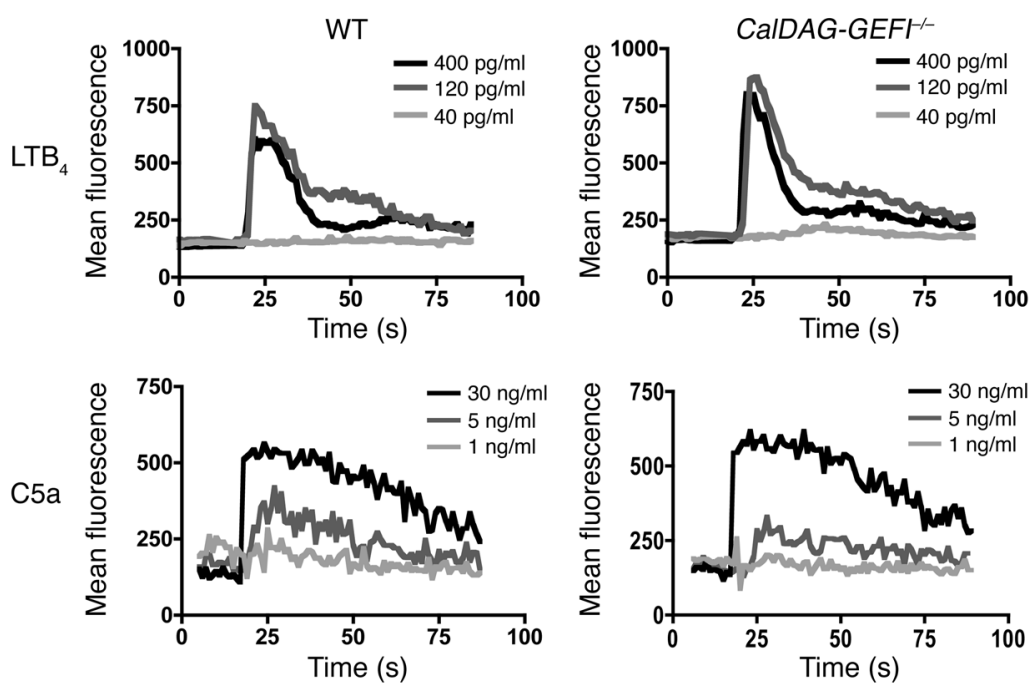

Figure 1

Degranulation, calcium flux, and ROS production in stimulated neutrophils. (A) Mac-1 expression. PBLs from WT and CaIDAG-GEFI-/- mice were kept resting or were activated for 10 minutes with $\mathrm{LTB}_{4}(300 \mathrm{nM}), \mathrm{C} 5 \mathrm{a}(50 \mathrm{ng} / \mathrm{ml})$, or PMA $(200 \mathrm{nM})$, stained with antibodies against Mac-1, and immediately analyzed by flow cytometry. $n=6$. (B) Calcium flux. PBLs from WT and CalDAG-GEFl-- mice were loaded with the calcium-sensitive dye Fluo-3, activated with the indicated doses of $\mathrm{LTB}_{4}$ or $\mathrm{C} 5 \mathrm{a}$, and immediately analyzed by flow cytometry. Results are representative of 6 individual experiments. (C) ROS formation. PBLs from WT and CaIDAG-GEFl-1- mice were loaded with the ROS-sensitive agent DCFDA, activated with PMA $(2 \mu \mathrm{M})$ or $\mathrm{MLP}(5 \mu \mathrm{M})$ for 30 minutes at $37^{\circ} \mathrm{C}$, and immediately analyzed by flow cytometry. $n=5$. ROS production is expressed as fold increase of mean fluorescence intensity over unstimulated cells. No significant differences in Mac-1 expression, calcium flux, or ROS production were observed between WT and CaIDAG-GEFI-deficient neutrophils. 


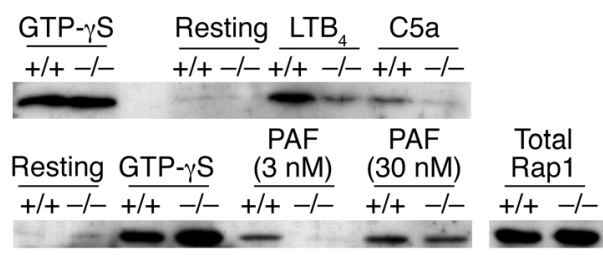

\section{Figure 2}

Impaired Rap1 activation in CaIDAG-GEFI-deficient neutrophils. Western blots of affinity-precipitated Rap1-GTP showing strongly decreased Rap1 activation in CalDAG-GEFI-deficient neutrophils (-/-) stimulated with $\mathrm{LTB}_{4}$ (300 nM for 30 seconds), C5a ( $75 \mathrm{ng} / \mathrm{ml}$ for 30 seconds), or PAF ( 3 or $30 \mathrm{nM}$ for 30 seconds) relative to that of WT neutrophils $(+/+)$. Equivalent loading of GTP onto Rap1 in neutrophils from WT and CaIDAG-GEFl-1- mice was shown by preincubation of lysates with GTP- $\gamma$ S. Total Rap1 levels were determined in whole cell lysates of WT and CalDAG-GEFI-deficient neutrophils. Results are representative of 3 individual experiments.

that the expression and function of agonist receptors and intracellular signaling molecules upstream of calcium flux are normal in CalDAG-GEFI-deficient neutrophils and that cellular functions such as degranulation and ROS formation are also intact.

CalDAG-GEFI mediates Rap1 activation in stimulated neutrophils. After establishing that agonist-triggered events upstream of calcium flux were normal in CalDAG-GEFI-deficient neutrophils, we next studied Rap1 activation in these cells. Within seconds, stimulation of leukocytes triggers the activation of Rap1, i.e., the exchange of GDP for GTP bound to the small GTPase $(23,24)$. We detected trace amounts of activated Rap1 in lysates of resting WT and CalDAG-GEFI-deficient neutrophils. Upon stimulation with $\mathrm{LTB}_{4}, \mathrm{C} 5 \mathrm{a}$, or a low dose of platelet activating factor (PAF; $3 \mathrm{nM}$ ), a strong increase in Rap1GTP was observed in lysates from WT but not CalDAG-GEFI-deficient neutrophils (Figure 2). The defect in PAF-induced activation of Rap1 observed in CalDAG-GEFI-deficient neutrophils was partially overcome by increasing the concentration of the agonist to $30 \mathrm{nM}$. Furthermore, we precipitated Rap1-GTP from lysates preincubated with GTP- $\gamma$ S, a form of GTP that cannot be hydrolyzed to GDP by the intrinsic GTPase activity of Rap1. We detected equivalent levels of GTP- $\gamma$ S-loaded Rap1 in mutant and WT samples, demonstrating that Rap1 from the neutrophils of CalDAG-GEFI--- mice did not have an intrinsic activation defect. The levels of total Rap1 were comparable in whole cell lysates from WT and CalDAG-GEFI-deficient neutrophils (Figure 2). Together, these data demonstrate that CalDAG-GEFI plays an important role in Rap1 activation in neutrophils stimulated by various agonists and that the defect in Rap1 activation observed in CalDAG-GEFI-deficient neutrophils may be overcome by increasing the concentration of the agonist.

Impaired activation of $\beta_{1}$ and $\beta_{2}$ integrins in CalDAG-GEFI-deficient neutrophils in vitro. To determine whether CalDAG-GEFI deficiency also affected integrin activation in neutrophils, we first studied $\beta_{1}$ integrin-mediated adhesion of isolated CalDAG-GEFI-deficient neutrophils to fibronectin. Neutrophils isolated from the bone marrow of WT and CalDAG-GEFI ${ }^{-1-}$ mice were activated with $300 \mathrm{nM}$ $\mathrm{LTB}_{4}$, and adhesion to fibronectin-coated plates was monitored. As shown in Figure 3A, adhesion of CalDAG-GEFI-deficient neutrophils was significantly impaired compared with that of WT neutrophils. We next examined $\beta_{2}$ integrin-mediated adhesion to fibrinogen, as it was shown previously that leukocyte binding to this ligand is mediated by the $\beta_{2}$ integrin receptors $\alpha_{M} \beta_{2}$ (Mac-1) and $\alpha_{X} \beta_{2}$ (25). Compared with WT neutrophils, CalDAG-GEFIdeficient neutrophils activated with $\mathrm{LTB}_{4}$ or PAF showed impaired adhesion to fibrinogen (Figure 3B).

CalDAG-GEFI is critical for $\beta_{2}$ integrin-mediated firm adhesion of neutrophils in vivo. We next examined the adhesion of leukocytes to activated mesenteric venules in the CalDAG-GEFI-- and WT mice. Venules were superfused with $300 \mathrm{nM} \mathrm{LTB}_{4}$ in PBS, and rolling as well as firmly adherent rhodamine 6G-labeled leukocytes were counted over a period of 20 minutes. Significantly more rolling leukocytes were observed in CalDAG-GEFI-/- than in WT mice $(P<0.04$; Figure 4A). However, this may not be a result of increased adhesiveness of the mutant cells, but rather the approximately 2-fold greater number of peripheral neutrophils circulating in these animals (15). It is not surprising that CalDAG-GEFI-deficient leukocytes rolled normally along stimulated venules, as this process depends largely on the binding of leukocyte PSGL-1 to endothelial selectins (26), and PSGL-1 is a constitutively active receptor that we found to be expressed normally in CalDAG-GEFI ${ }^{-/-}$mice (Table 1). In contrast, firm leukocyte adhesion induced by $\mathrm{LTB}_{4}$ requires the rapid activation of integrins $(27,28)$ via intracellular signaling pathways. We found that WT leukocytes adhered firmly to mesenteric venules within minutes of $\mathrm{LTB}_{4}$ superfusion, while firm adhesion of
A

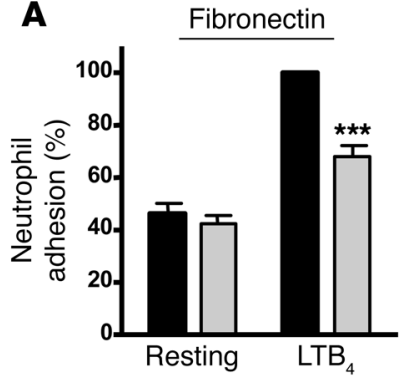

B

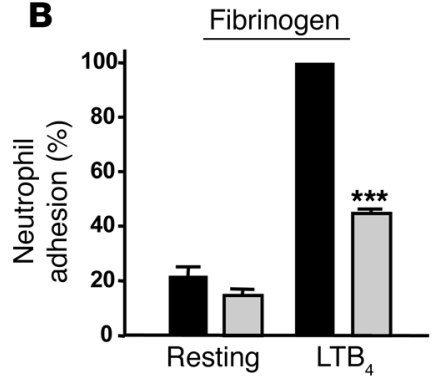

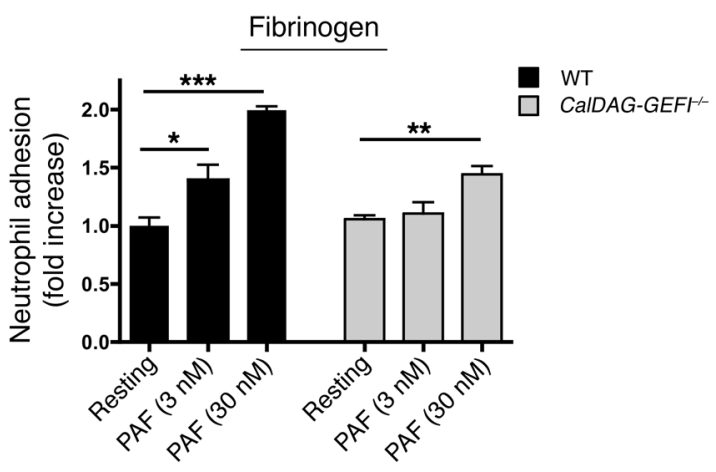

Figure 3

CalDAG-GEFI deficiency causes impaired $\beta_{1}$ integrin- and $\beta_{2}$ integrin-mediated adhesion of neutrophils in vitro. (A and $\left.\mathbf{B}\right)$ Neutrophil adhesion to fibronectin (A) or fibrinogen (B) in vitro. WT or CalDAG-GEFI-deficient neutrophils isolated from bone marrow were added to fibronectin- or fibrinogen-coated plates and incubated for 30 minutes in the presence or absence (i.e., resting) of $300 \mathrm{nM} \mathrm{LTB} 4$ or 3 or $30 \mathrm{nM}$ PAF. Plates were washed and adherent neutrophils were counted. $n=4$. ${ }^{*} P<0.05,{ }^{* \star} P<0.01,{ }^{* *} P<0.001$. 
A

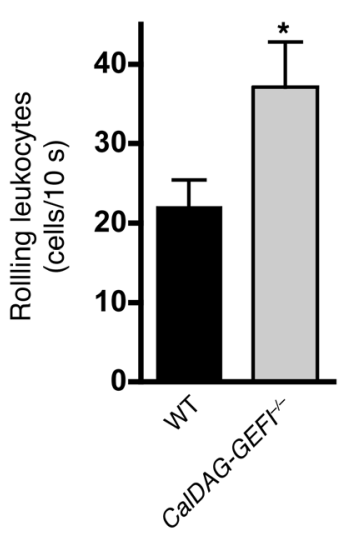

B

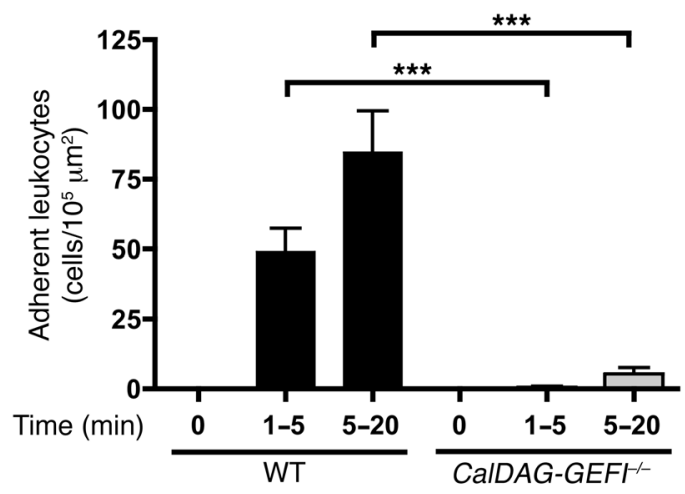

C

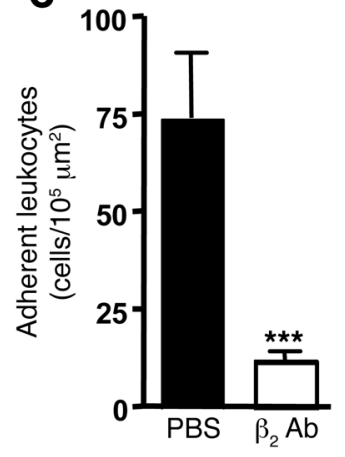

\section{Figure 4}

Impaired firm adhesion of leukocytes to mesenteric venules in CaIDAG-GEFl-1- mice. (A) Leukocyte rolling in vivo. The number of rolling leukocytes in WT or CaIDAG-GEFl-l- mice was determined by intravital microscopy. Mice were infused with rhodamine $6 \mathrm{G}$ to label circulating leukocytes. Leukocyte rolling was quantified 1-5 minutes after superfusion with $300 \mathrm{nM} \mathrm{LTB}$. (B) Firm adhesion. WT or CalDAG-GEFI-deficient leukocytes were considered firmly adherent when they remained stationary for more than 30 seconds. $n=6$. (C) Blocking antibodies to $\beta_{2}$ integrin

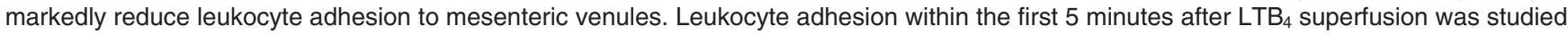
in WT mice infused with PBS or $40 \mu \mathrm{g}$ anti- $\beta_{2}$ antibodies $\left(\beta_{2} \mathrm{Ab}\right) . n=3 .{ }^{*} P<0.05$, ${ }^{* * *} P<0.001$. No significant difference in leukocyte adhesion was observed between untreated and control IgG-treated WT mice (not shown).

CalDAG-GEFI-deficient leukocytes was reduced by more than $90 \%$ (Figure 4B). Infusion of WT mice with a blocking antibody against $\beta_{2}$ integrins reduced the firm adhesion of leukocytes by more than $80 \%$ (Figure $4 \mathrm{C}$ ), confirming the important role of $\beta_{2}$ integrins in leukocyte adhesion previously shown in venules of $C D 18^{-/-}(29)$ and $\mathrm{Mac1}^{-/-}$mice (28). Our results indicate that CalDAG-GEFI is needed for the activation of $\beta_{2}$ integrins both in vitro and in vivo.

Impaired inflammatory response in CalDAG-GEFI'-- mice. To test the ability of CalDAG-GEFI-/- mice to respond to acute inflammation, we challenged WT and mutant mice by intraperitoneal injection of thioglycollate (TG). Using this model, several groups have shown a key role for $\beta_{2}$ integrins in the rapid recruitment of neutrophils to the peritoneal cavity (30-32). Others did not find a marked reduction in the total number of neutrophils recruited to the peritoneum of $\mathrm{CD} 18^{-/-}$mice (7). However, $C D 18^{-/-}$mice are characterized by neutrophil counts elevated by greater than 10 -fold compared with controls, suggesting that the efficiency of TG-induced neutrophil recruitment was also impaired in these studies. We found few neutrophils in the unchallenged peritoneal cavities of WT and CalDAG-GEFI--- mice (Figure 5A). In WT mice, we found robust intraperitoneal infiltration of neutrophils 4 hours after challenge. In contrast, CalDAG-GEFI ${ }^{-/-}$mice exhibited approximately $80 \%$ reduction in neutrophil infiltration compared with WT mice after challenge $(P<0.001$; Figure 5A), demonstrating a pivotal function for CalDAG-GEFI in neutrophil recruitment to inflamed peritoneum.

To test CalDAG-GEFI'-/- mice in a specifically $\beta_{2}$ integrin-dependent inflammation model (7), we studied neutrophil infiltration into croton oil-irritated ears. As shown in Figure 5B, the number of neutrophils counted in the croton oil-painted ears of CalDAG-GEFI--mice was about $60 \%$ that of WT controls $(P<0.001)$. These findings strengthen the case for CalDAG-GEFI as a critical intracellular signaling molecule upstream of $\beta_{2}$ integrin activation.

Impaired $\beta_{1}$ integrin activation in CalDAG-GEFI-deficient platelets. To determine whether CalDAG-GEFI also regulates $\beta_{1}$ integrin func- tion in platelets, we examined platelet adhesion to established substrates of $\beta_{1}$ integrins. Platelets express various members of the $\beta_{1}$ integrin subfamily, including $\alpha_{2} \beta_{1}$ (collagen as the main ligand), $\alpha_{5} \beta_{1}$ (fibronectin as the main ligand), and $\alpha_{6} \beta_{1}$ (laminin as the main ligand) $(33,34)$. The expression levels of $\beta_{1}$ integrins were comparable in platelets from CalDAG-GEFI ${ }^{-1}$ and WT mice (mean fluorescence intensity, $240.5 \pm 8.0$ and $242.7 \pm 14.8$, respectively). We studied the adhesion of activated WT and CalDAG-GEFIdeficient platelets to a laminin-coated surface by stimulating platelets with PAR4-activating peptide (PAR4p; GYPGKF) or a combination of $\mathrm{ADP}$ and the thromboxane $\mathrm{A}_{2}$ mimetic U46619. In order to avoid adhesion/aggregation of platelets mediated by $\alpha_{\mathrm{IIb}} \beta_{3}$ integrin, the experiments were performed in the presence of a blocking antibody against this receptor (35). In contrast to activated WT platelets, which showed robust adhesion to laminin, the adhesion of CalDAG-GEFI-deficient platelets stimulated with either agonist was significantly inhibited (Figure 6A). The dependence of the adhesion process on $\alpha_{6} \beta_{1}$ integrin was demonstrated by blocking this receptor on WT platelets (Figure 6A). With the same experimental setup, we also tested the adhesion of CalDAG-GEFIdeficient platelets to fibronectin, a process mediated by integrins $\alpha_{5} \beta_{1}$ and $\alpha_{\mathrm{II}} \beta_{3}(34,36)$. Because we are not aware of any specific reagents that inhibit $\alpha_{\mathrm{IIb}} \beta_{3}$ integrin-mediated adhesion of mouse platelets to fibronectin, we used EDTA in control experiments to inhibit both $\beta_{1}$ integrin- and $\beta_{3}$ integrin-mediated adhesion. The specificity of the adhesion process for $\beta_{3}$ and $\beta_{1}$ integrins was verified in studies with activated platelets lacking $\beta_{3}$ integrins, which adhered to fibronectin in an $\alpha_{5} \beta_{1}$ integrin-dependent manner (data not shown). Again, U46619/ADP-stimulated CalDAG-GEFIdeficient platelets showed significantly impaired adhesion to fibronectin (Figure 6B). No significant difference in the adhesion of WT and CalDAG-GEFI-deficient platelets stimulated with PAR4 $p$ was observed, indicating that 1 or more alternative signaling pathways mediated $\beta_{1}$ integrin activation in platelets stimulated by PAR4. These results provide strong evidence that 

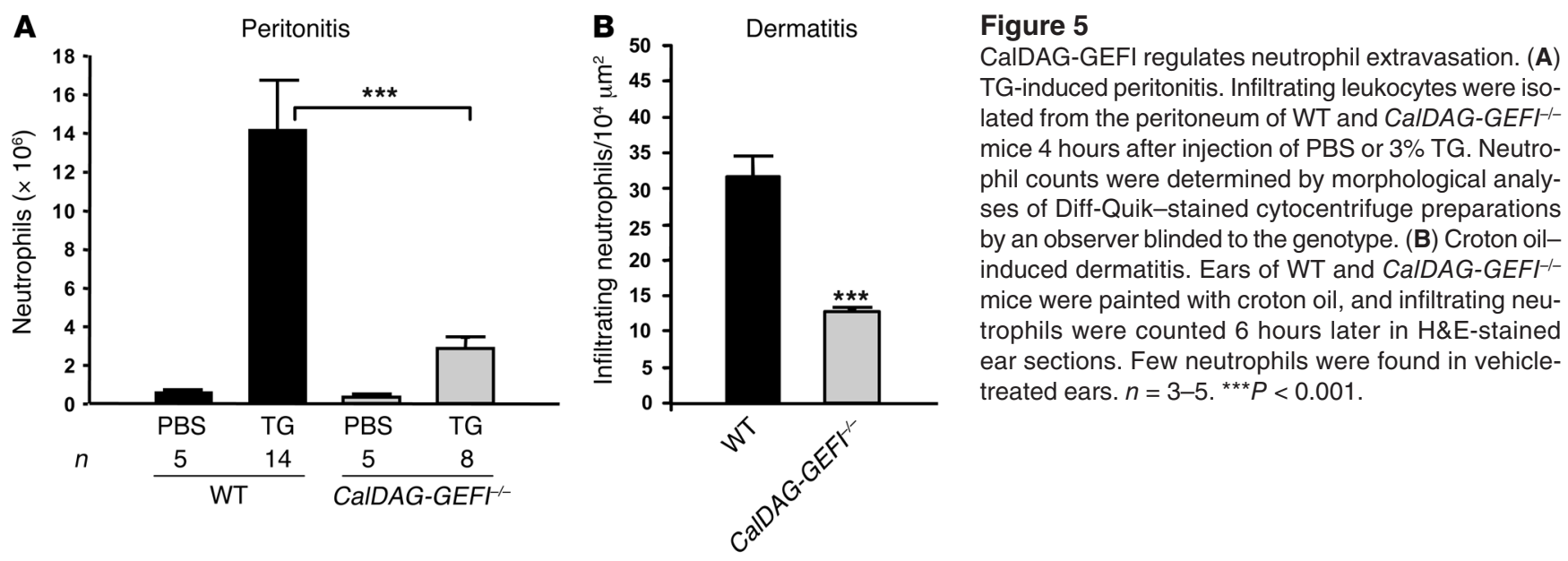

CalDAG-GEFI is an important signaling molecule mediating activation of $\alpha_{6} \beta_{1}$ and $\alpha_{5} \beta_{1}$ integrins in platelets activated by some, but not all, platelet agonists.

CalDAG-GEFI ${ }^{-1-}$ mice do not form thrombi in an experimental arterial thrombosis model. To determine the effect of defective $\beta_{1}$ and $\beta_{3}$ integrin activation on thrombus formation in vivo, we studied WT and CalDAG-GEFI ${ }^{-1-}$ mice in a model of $\mathrm{FeCl}_{3}$-induced arterial thrombosis $(37,38)$. In this model, $\mathrm{FeCl}_{3}$ causes endothelial denudation leading to the exposure of extracellular matrix, which in turn promotes the adhesion and activation of circulating platelets. We did not observe a significant difference between WT and CalDAG-GEFI ${ }^{-/-}$mice in the frequency of platelet tethering (Figure 7, A and B). This was not surprising, as the initial tethering of platelets depends on the constitutively active GPIb-V-IX receptor complex (33), which was expressed in high copy numbers on both WT and mutant platelets (mean fluorescence intensity, $\mathrm{CalDAG}-\mathrm{GEFI}^{-/-}$, $907 \pm 37.8$; WT, $886.7 \pm 35.9)$. Firm adhesion and/or aggregation of platelets at sites of vascular injury, however, requires agonist-induced inside-out activation of platelet integrins. The first thrombi formed in WT mice approximately 10 minutes after application of $\mathrm{FeCl}_{3}$ (data not shown), and vessel occlusion was observed in all WT mice within 20 minutes (mean occlusion time,
$14 \pm 1.3$ minutes), whereas thrombus formation was completely absent in CalDAG-GEFI ${ }^{-1}$ mice for the duration of the 40-minute observation period (Figure 7, A and C). Video of real-time platelet adhesion/thrombus formation in injured arterioles is provided as supplemental data (Supplemental Video 1; available online with this article; doi:10.1172/JCI30575DS1). Thus, CalDAG-GEFI is a major regulator of $\beta_{1}$ and $\beta_{3}$ integrin activation, and its absence has a profound impact on platelet function in vivo.

\section{Discussion}

We found that CalDAG-GEFI was a major regulator of the activation of $\beta_{1}, \beta_{2}$, and $\beta_{3}$ integrins on platelets and neutrophils both in vitro and in vivo. As a consequence, mice lacking CalDAG-GEFI had defective inflammatory responses and markedly impaired ability to form thrombi in response to vascular injury. This phenotype is strikingly similar to that described for LAD-III patients, which combines a mild LAD with a Glanzmann-like bleeding disorder (9-13). In all cases reported to date, the clinical symptoms of LAD-III seem to be caused by a defect in the activation, but not the expression or structure, of $\beta_{1}, \beta_{2}$, and $\beta_{3}$ integrins on leukocytes and platelets (9). The successful treatment of LAD-III patients by bone marrow transplantation strongly suggests that the genetic defect
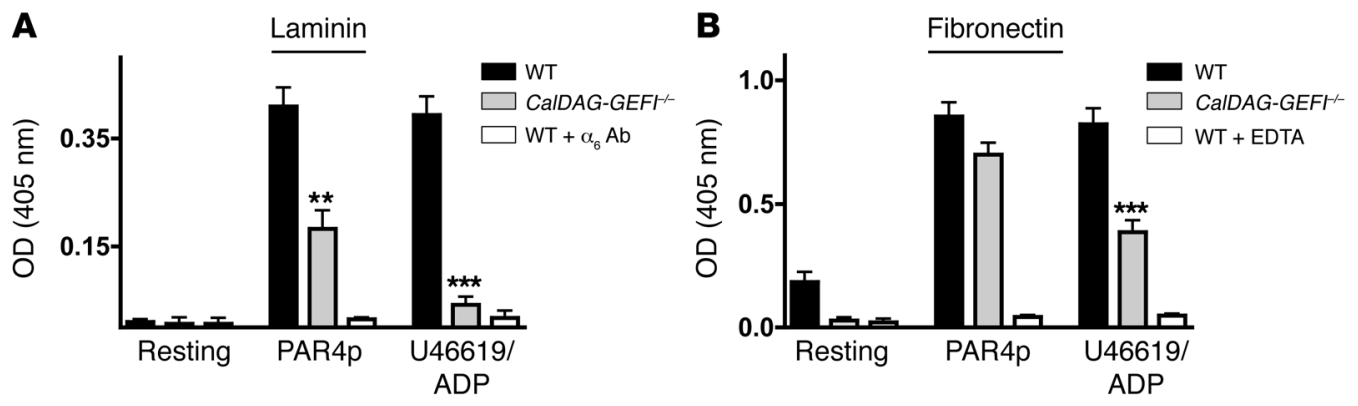

\section{Figure 6}

Impaired activation of $\beta_{1}$ integrins in CaIDAG-GEFI-deficient platelets. (A and B) Biotinylated WT and CalDAG-GEFI-deficient platelets were stimulated with PAR4p (2 mM) or U46619/ADP $(5$ or $10 \mu \mathrm{M})$ in the presence of a blocking antibody to $\alpha_{\| 1 b} \beta_{3}$ and allowed to adhere for 30 minutes under static conditions to laminin (A) or fibronectin (B) in microtiter plates. A separate group of WT platelets was pretreated with a blocking antibody to $\alpha_{6}$ (adhesion to laminin) or with EDTA (adhesion to fibronectin) to demonstrate the integrin dependency of the adhesion process. Adherent platelets were quantified colorimetrically for peroxidase activity. Data are mean \pm SEM of 3 individual experiments in triplicate wells. ${ }^{\star \star} P<0.01,{ }^{\star \star *} P<0.001$. Similar results were observed with nonbiotinylated platelets when the number of adhesive platelets was determined by light microscopy (not shown). 
A

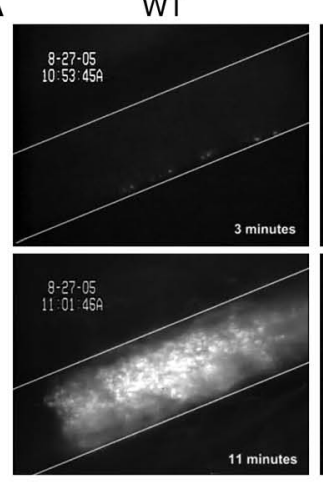

CaIDAG-GEFI-

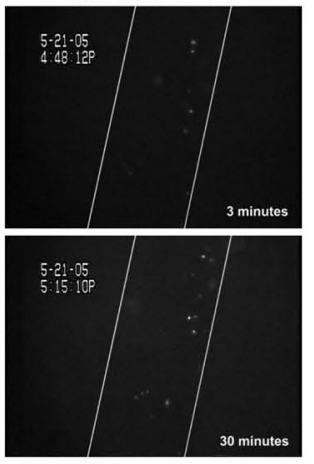

B

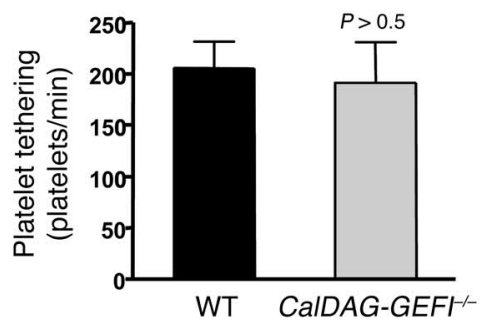

C

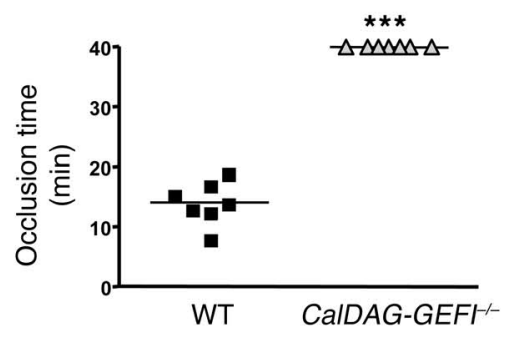

Figure 7

Platelet adhesion and thrombus formation in $\mathrm{FeCl}_{3}$-injured arterioles. (A) WT and CalDAG-GEFI-- mice were injected with calcein-green-labeled platelets of the respective genotype, and platelet adhesion was monitored in mesenteric arterioles (diameter, WT, $90.7 \pm 3.5 \mu \mathrm{m}$; CalDAG-GEFI-/, $96.4 \pm 4.0 \mu \mathrm{m}$ ) upon application of $\mathrm{FeCl}_{3}$. Images show platelet adhesion and thrombus formation in arterioles at the indicated times after application of $\mathrm{FeCl}_{3}$. The direction of blood flow is from top to bottom. (B and $\mathbf{C}$ ) Comparison of the number of tethering platelets (B) and the time of occlusion (C) in $\mathrm{FeCl}_{3}$-treated mesenteric arterioles of WT and CalDAG-GEFl-/- mice. No thrombi formed in the mutant mice during the 40-minute observation period. ${ }^{* *} P<0.001$

underlying the disease resides in hematopoietic cells (9). Thus, it has been speculated that the absence or malfunction of a key integrin regulator expressed in hematopoietic cells is responsible for the phenotype observed in LAD-III patients (9). We propose here that CalDAG-GEFI is such a regulator and that genetic deficiency of CalDAG-GEFI may be present in patients with LAD-III.

Like leukocytes from LAD-III patients (10-12), neutrophils from CalDAG-GEFI-/ mice behaved normally in many functional assays including ROS formation, intracellular calcium flux, and granule release (Figure 1), demonstrating that the cells were fully capable of activating intracellular signaling pathways. CalDAG-GEFIdeficient neutrophils expressed normal levels of $\beta_{2}$ integrins and PSGL-1 on the cell surface, while the surface expression of $\beta_{1}$ integrins and L-selectin was significantly reduced (Table 1). The decrease in L-selectin expression was similar to that previously observed in $\mathrm{P} / \mathrm{E}$-selectin-deficient mice, which also show a defect in neutrophil extravasation (20). Thus, it may reflect shedding of the receptor from activated blood leukocytes that are unable to extravasate into tissue. Interestingly, reduced L-selectin expression was also reported for leukocytes from 1 LAD-III patient (13). As a consequence of their defective integrin activation, CalDAG-GEFIdeficient neutrophils showed an impaired response to acute inflammation. Compared with controls, significantly fewer CalDAG-GEFI-deficient neutrophils migrated into the inflamed tissues of mice subjected to experimental peritonitis or dermatitis (Figure 5). It is interesting to note, however, that neutrophil recruitment in the dermatitis model was only partially reduced in CalDAG-GEFI'-- mice (approximately $40 \%$ of control), while previous studies showed a complete inhibition in neutrophil recruitment in this model in $C D 18^{-1-}$ mice (7). These data suggest that CalDAG-GEFI plays an important and specific role in the activation of $\beta_{2}$ integrins in neutrophils and that, at least in some inflammatory situations, other signaling molecules such as PKC family members $(39,40)$ or other Rap-GEFs $(41)$ can serve as alternative pathways leading to $\beta_{2}$ integrin activation in the absence of CalDAG-GEFI. Similarly, we have previously shown robust aggregation of CalDAG-GEFI-deficient platelets stimulated with thrombin or collagen (15). In further studies, we iden- tified signaling by PKC as an independent pathway allowing for $\alpha_{\text {IIb }} \beta_{3}$ activation in the absence of CalDAG-GEFI (Bergmeier et al., unpublished observations).

We now provide evidence that CalDAG-GEFI was also critical for the activation of platelet $\beta_{1}$ integrins (Figure 6). Activation of $\beta_{1}$ integrins was almost completely inhibited in CalDAG-GEFI-deficient platelets stimulated with ADP and the thromboxane $\mathrm{A}_{2}$ analog $\mathrm{U} 46619$, while it was only partially inhibited in CalDAG-GEFI-deficient platelets activated by PAR4 receptors (Figure 6). Our results confirm observations by Gruner et al., who showed that $\beta_{1}$ integrins are expressed on resting mouse platelets in a low-affinity state and that cellular activation is required for these integrins to shift to a high-affinity state (34). Previous work with human platelets suggested that integrins $\alpha_{5} \beta_{1}$ and $\alpha_{6} \beta_{1}$ may be expressed constitutively in a high-affinity state, as these cells spontaneously adhere to fibronectin and laminin, respectively $(36,42)$. However, these studies only analyzed the adhesion of unstimulated platelets and did not investigate whether platelet activation would further increase the number of adherent cells. Based on our findings that strong agonists induced significant activation of $\beta_{1}$ and $\beta_{3}$ integrins in knockout platelets in vitro, we were surprised that CalDAG-GEFI deficiency led to complete inhibition of arterial thrombus formation (Figure 7). Probably the simplest explanation for this finding is that the collagen and thrombin concentrations that a platelet encounters at sites of vascular damage are much lower than those used in our in vitro studies. Alternatively, the strong defect in platelet adhesion observed in CalDAG-GEFI ${ }^{-/}$mice could reflect the critical role that CalDAG-GEFI plays in outside-in signaling by ligand-occupied integrins in platelets (43) or that CalDAG-GEFIdeficient platelets are not capable of activating their integrins on the subsecond scale required for successful adhesion under conditions of flow. Such a defect has been suggested for leukocytes from LAD-III patients, which showed a prominent adhesion deficiency under flow (13). We similarly found the most striking defects in the adhesion of CalDAG-GEFI-deficient neutrophils in vivo rather than in vitro under static conditions (Figures 3 and 4).

Although we observed strong defects in platelet and neutrophil adhesion in CalDAG-GEFI ${ }^{-/}$mice, we did not observe spontaneous bleeding or apparent infections in these mice. These findings may 
appear in contrast to the phenotype described for patients with LAD-III, who have mild to severe bleeding complications as well as recurrent infections (9). However, in patients, bleeding or infection is most likely caused by accidental tissue damage or exposure to pathogens, challenges that mice held in a restricted, pathogen-free living space hardly encounter. Spontaneous bleeding complications are also rare in mouse models of Bernard-Soulier syndrome or severe thrombocytopenia, while strongly impaired hemostasis is observed in tail bleed assays in these mice $(44,45)$.

The small GTPase Rap1 has recently been identified as a critical molecule regulating integrin activation in many cell types, including platelets, megakaryocytes, and neutrophils $(23,24)$. However, the widespread expression of Rap 1 and its reported importance in nonhematopoietic cells $(23,24)$ make it unlikely that Rap1 itself is mutated in patients with LAD-III (9). In addition, a recent study by Kinashi et al. demonstrated normal expression but impaired activation of Rap1 in transformed PBLs derived from a patient with LAD-III (46). The authors concluded that a Rap1-GEF activity essential for Rap1 and integrin activation was defective in these cells. We have shown that CalDAG-GEFI is a major regulator of Rap1 and $\beta_{1}$ and $\beta_{3}$ integrin activation in platelets (Figure 6 and ref. 15) and that it is also critical for activation of Rap1 (Figure 2) and $\beta_{1}$ and $\beta_{2}$ integrin (Figures 3 and 4 ) in neutrophils. In addition, the expression of CalDAG-GEFI is much more tissue specific than that of Rap1 $(15,17)$, which may explain why mice lacking CalDAG-GEFI do not show obvious defects during embryonic development, whereas mice lacking Rap1b display approximately $85 \%$ embryonic lethality (47). Thus, CalDAG-GEFI could be the defective Rap-GEF in platelets and leukocytes of LAD-III patients.

Other proteins involved in the inside-out activation of more than 1 integrin family include cytoskeletal proteins such as talin or filamin $(48,49)$, integrin-linked kinase (ILK) $(50)$, or members of the PKC family $(39,40)$. These proteins, however, are not restricted to cells of the hematopoietic lineage; thus, mutations in the genes encoding them are not likely responsible for LAD-III. Furthermore, normal expression of various cytoskeletal adapters, PKC isoforms, and ILK were reported in leukocytes from 1 patient with LAD-III (12).

Different phenotypes have been described in individual patients with LAD-III (10-13), which suggests that there are variations in disease penetrance or that more than 1 gene may be implicated. For example, Harris et al. (11) found that a patient's integrins had an intrinsic defect in their avidity to bind ligands that could not be overcome by activating antibodies or exogenous cations and that neutrophils from this patient showed an impaired chemotactic response. Such defects have not to our knowledge been reported by other groups. Alon et al. (13) found that leukocytes from another patient with LAD-III exhibited impaired adhesion under physiological flow conditions, but that their adhesion under static conditions was similar to that of controls. Adhesion under static conditions has been found to be severely impaired in all other reported cases of LAD-III (10-12). In CalDAG-GEFI-/- mice, the defect in neutrophil adhesion was most prominent under conditions of flow, but it was also observed under static conditions.

Our finding that CalDAG-GEFI was critical to the activation of $\beta_{1}, \beta_{2}$, and $\beta_{3}$ integrins in mouse platelets and neutrophils suggests this gene may be defective in patients with LAD-III. With their defects in thrombus formation and leukocyte recruitment to sites of inflammation, mice deficient in CalDAG-GEFI represent an animal model for this disease.

\section{Methods}

\section{Mice}

CalDAG-GEFI ${ }^{--}$(15) and littermate control WT mice were obtained from the mouse facility at MIT and were bred in the mouse facility of the CBR Institute for Biomedical Research. Itgb3 $3^{-/-}$mice on a BALB $/ \mathrm{c}$ background were a generous gift from R. Hynes (MIT). Experimental procedures were approved by the Animal Care and Use Committee of the CBR Institute for Biomedical Research.

\section{Flow cytometry}

Blood was drawn from the retroorbital plexus into heparinized tubes. One milliliter of whole blood was incubated in $10 \mathrm{ml}$ of red blood cell lysis buffer (155 $\mathrm{mM} \mathrm{NH}_{4} \mathrm{Cl}, 10 \mathrm{mM} \mathrm{KHCO}_{3}, 0.1 \mathrm{mM} \mathrm{Na}_{2}$ EDTA, pH 7.4) for 10 minutes and then centrifuged for 8 minutes at $185 \mathrm{~g}$. The supernatant was discarded, and the pellet resuspended in a modified Tyrode buffer (15).

Expression of adhesion receptors on resting PBLs. Resting cells were stained with fluorescently labeled antibodies for 30 minutes at $4{ }^{\circ} \mathrm{C}$ and immediately analyzed by flow cytometry. Neutrophils were identified by determining forward/side scatter characteristics and staining with the neutrophilspecific monoclonal antibody Gr-1 (BD Biosciences).

Mac-1 expression on resting and activated neutrophils. PBLs were kept resting or were stimulated with $300 \mathrm{nM} \mathrm{LTB}_{4}, 200 \mathrm{nM} \mathrm{PMA}$, or $50 \mathrm{ng} / \mathrm{ml} \mathrm{C5a}$ for 10 minutes at room temperature. Cells were stained with $2 \mu \mathrm{g} / \mathrm{ml}$ FITC-conjugated anti-Mac-1 (BD Biosciences) and were immediately analyzed by flow cytometry.

Calcium flux measurements in peripheral blood neutrophils. PBLs were incubated with $5 \mu \mathrm{M}$ of the calcium-sensing dye Fluo-3 (Invitrogen) for 15 minutes, washed in modified Tyrode buffer containing $1 \mathrm{mM} \mathrm{CaCl}_{2}$ and $1 \mathrm{mM} \mathrm{MgCl}_{2}$, activated with the indicated agonists, and analyzed immediately by FACS. Data were analyzed with FlowJo software (version 6.4.3; Tree Star Inc.).

Production of ROS in neutrophils. PBLs were resuspended in RPMI and incubated with $7.5 \mu \mathrm{M}$ ROS-sensing dye DCFDA-H2 (Invitrogen) for 15 minutes. After spinning labelled cells at $185 \mathrm{~g}$ for 5 minutes, excessive dye was discarded and cells were resuspended in RPMI buffer. PBS, $5 \mu \mathrm{M}$ fMLP, or $2 \mu \mathrm{M}$ PMA was added to DCFDA-loaded PBLs at time point 0. Mean fluorescence values in channel 1 (FL1) of approximately 2,000 Gr-1-positive cells were assessed in 5-minute intervals for up to 60 minutes. To normalize individual experiments, the ratio between mean FL1 values of stimulated and unstimulated cells was determined.

\section{Rap1 activation}

Amounts of activated Rap 1 in neutrophils were measured using a protocol similar to the one previously described for platelets (15). Neutrophils were isolated from bone marrow of WT and CalDAG-GEFI ${ }^{-/-}$mice using negative sorting with MACS separation columns (Miltenyi Biotec). After red cell lysis, bone marrow cells were incubated for 10 minutes at $4^{\circ} \mathrm{C}$ with $10 \mu \mathrm{g} / \mathrm{ml}$ anti-CD45R, $10 \mu \mathrm{g} / \mathrm{ml}$ anti-CD5, $5 \mu \mathrm{g} / \mathrm{ml}$ anti-CD8, and $5 \mu \mathrm{g} / \mathrm{ml}$ anti-CD4 (all from BD Biosciences). After 2 washing steps, cells were incubated with anti-rat Ig beads for 10 minutes at $4{ }^{\circ} \mathrm{C}$, washed again, and run through $25 \mathrm{LD}$ magnetic columns (Miltenyi Biotec). The eluted cells were stained with Gr-1 antibody, and the percentage of Gr-1-positive cells was determined by flow cytometry. Only samples containing more than $90 \%$ Gr-1-positive cells were used for adhesion studies. Neutrophils $\left(5 \times 10^{6}\right)$ were activated for 30 seconds with $300 \mathrm{nM} \mathrm{LTB}_{4}, 75 \mathrm{ng} / \mathrm{ml} \mathrm{C5a}$, or 3 or $30 \mathrm{nM}$ PAF and immediately lysed with ice-cold lysis buffer $(25 \mathrm{mM}$ Tris- $\mathrm{HCl}$, $\mathrm{pH} 7.5,150 \mathrm{mM} \mathrm{NaCl}, 1 \%$ Nonidet P-40, $5 \mathrm{mM} \mathrm{MgCl}_{2}, 5 \%$ glycerol, and complete protease inhibitor cocktail lacking EDTA; Roche Diagnostics). Detection of activated Rap1 (Rap1-GTP) in neutrophil lysates was performed according to the instructions of the manufacturer (Pierce). 
Briefly, Rap1-GTP (or Rap1-GTP- $\gamma$ S) was precipitated from lysates using a GST-RalGDS-RBD fusion protein. Precipitated proteins were separated on a $15 \%$ SDS-PAGE gel and transferred to PVDF membranes (Millipore). Rap1 was detected with rabbit polyclonal antibodies followed by anti-rabbit antibodies conjugated to horseradish peroxidase (Vector Laboratories). Immunoreactivity was detected by Western Lightning enhanced chemiluminescence (PerkinElmer Life Sciences).

To determine the level of total Rap $1,1 \times 10^{6}$ WT or CalDAG-GEFI-deficient neutrophils were lysed with reducing SDS sample buffer, and proteins were separated on a 15\% SDS-PAGE gel. Rap1 was detected with rabbit polyclonal antibodies as described above.

\section{Neutrophil adbesion assay}

Ninety-six-well plates (Costar) were coated with $2 \mathrm{mg} / \mathrm{ml}$ fibrinogen or $10 \mu \mathrm{g} / \mathrm{ml}$ fibronectin (both from Sigma-Aldrich) overnight and blocked with $1 \%$ BSA for 2 hours. Isolated bone marrow neutrophils $\left(1 \times 10^{6} / \mathrm{ml}\right)$ were added in the presence or absence of the indicated agonists and incubated for 30 minutes at $37^{\circ} \mathrm{C}$. The supernatant was discarded, the plate was washed with PBS, and the neutrophils adhered to the plate were counted using a Nikon TMS microscope. Adherent neutrophils in the region of interest were counted by 2 individuals blinded to their source. Alternatively, neutrophil adhesion was analyzed by quantifying myeloperoxidase (MPO) activity in the lysate of adherent cells (51). Briefly, adherent neutrophils were lysed in a potassium phosphate buffer containing $0.5 \%$ hexadecyl trimethyl ammonium bromide (HTAB), and MPO activity was determined by adding tetramethylbenzidine (TMB) as a substrate. OD readings were performed in a 96-well microplate reader at a wavelength of $630 \mathrm{~nm}$.

\section{Platelet adhesion assay}

For platelet adhesion studies, 96-well plates (Nunc) were coated overnight at $4^{\circ} \mathrm{C}$ with either laminin or fibronectin (both from Sigma-Aldrich) at a concentration of $4 \mu \mathrm{g} / \mathrm{ml}$ and then incubated with PBS plus 5\% BSA for 2 hours at $37^{\circ} \mathrm{C}$. Platelet-rich plasma (PRP) was obtained from heparinized whole blood by centrifugation at $100 \mathrm{~g}$ for 10 minutes and then centrifuged at $700 \mathrm{~g}$ in the presence of $\mathrm{PGI}_{2}(2 \mu \mathrm{g} / \mathrm{ml})$ for 7 minutes at room temperature. After 2 washing steps, pelleted platelets were resuspended in modified Tyrode-HEPES buffer. Platelets $\left(2 \times 10^{9} / \mathrm{ml}\right)$ were incubated with NHS-biotin (1 mM; Pierce) for 10 minutes, washed twice, and resuspended in a modified Tyrode buffer containing $\mathrm{Ca}^{2+}$ and $\mathrm{Mg}^{2+}(1 \mathrm{mM}$ each). The cells were either left unstimulated or activated with $2 \mathrm{mM}$ PAR4p or 5 or $10 \mu \mathrm{M} \mathrm{U} 46619 / \mathrm{ADP}$ and immediately plated. After incubation for 30 minutes at $37^{\circ} \mathrm{C}$, the plate was rinsed 3 times with $100 \mu \mathrm{lBS}$, and $50 \mu \mathrm{l}$ of HRP-labeled streptavidin solution $(1 \mu \mathrm{g} / \mathrm{ml}$; Jackson ImmunoResearch Laboratories) was added to each well for 15 minutes. After extensive washing, $100 \mu \mathrm{l}$ HRP substrate (ABTS; Roche Diagnostics) was added to each well, and the OD at a wavelength of $405 \mathrm{~nm}$ was determined after $5 \mathrm{~min}$ utes. To inhibit $\beta_{3}$ integrin-mediated platelet aggregation, all experiments were performed in the presence of a blocking antibody to $\alpha_{\mathrm{IIb}} \beta_{3}(50 \mu \mathrm{g} / \mathrm{ml}$; emfret Analytics). Blocking antibodies to $\alpha_{6}$ integrin $(50 \mu \mathrm{g} / \mathrm{ml} ; \mathrm{BD}$ Biosciences - Pharmingen) or EDTA (15 mM) were used as inhibitors in adhesion studies of laminin and fibronectin, respectively. Calcium flux and integrin activation studies did not show significant differences between biotinylated and nonbiotinylated platelets (data not shown).

\section{Intravital microscopy}

Thrombosis model. Platelets were labeled for 10 minutes with calcein-green $(5 \mu \mathrm{g} / \mathrm{ml}$; Invitrogen) and infused into 3- to 5-week-old anesthetized male CalDAG-GEFI ${ }^{-/-}$mice or their littermate WT controls. The mesentery was exposed through a midline abdominal incision. Vessels with a shear rate of 1,000-1,500 s $\mathrm{s}^{-1}$ were selected by use of an Optical Doppler Velocimeter
(Cardiovascular Research Institute, Texas A\&M University System Health Science Center). Arterioles were examined with a Zeiss Axiovert 135 inverted microscope (Zeiss), and adhesion of fluorescently labeled platelets was monitored with a silicon-intensified tube black and white camera (C2400-08; Hamamatsu) connected to an S-VHS video recorder (AG-6730; Panasonic). Vessel injury was generated by placing a filter paper $(1 \times 4 \mathrm{~mm})$ soaked with $10 \% \mathrm{FeCl}_{3}$ over the vessel for 5 minutes. The filter paper was then removed, and the vessel was superfused with saline at $37^{\circ} \mathrm{C}$. Vessels were monitored for 40 minutes after $\mathrm{FeCl}_{3}$ treatment or until blood flow had stopped for longer than 10 seconds (identified as occlusion time).

Leukocyte adhesion. Male mice were infused with rhodamine 6G $(100 \mu \mathrm{g} / \mathrm{ml}$ in PBS) to label circulating leukocytes. Exposed mesenteric venules (shear rates ranging 50-150 s-1) were superfused with PBS containing $300 \mathrm{nM} \mathrm{LTB}_{4}$ to initiate leukocyte adhesion. We made 30 -second recordings of fluorescent cells in different parts of 2-4 venules per animal during 4 consecutive 5 -minute intervals. The number of rolling leukocytes was determined by counting the cells passing through a perpendicular plane in 10 seconds. To determine firm adhesion of cells, we counted the number of leukocytes that remained stationary for more than 30 seconds.

To study the role of $\beta_{2}$ integrins in leukocyte adhesion under these experimental conditions, mice were infused with $40 \mu \mathrm{g}$ inhibitory anti- $\beta_{2}$ antibodies (BD Biosciences) 15 minutes prior to the start of the experiment and analyzed as described above.

\section{Dermatitis}

Irritant dermatitis was induced by topical application of croton oil (7). Mice were anesthetized by isoflurane inhalation, and each side of 1 ear was treated with $10 \mu \mathrm{l}$ of $2 \%$ croton oil (Sigma-Aldrich) in 4:1 acetone/olive oil. After 6 hours, mice were sacrificed by an overdose of halothane inhalation. Ears were removed, fixed in $10 \%$ formalin, and embedded in paraffin, and sections were stained with $\mathrm{H} \& \mathrm{E}$ for examination by light microscopy.

\section{Peritonitis}

Mice were injected intraperitoneally with $1 \mathrm{ml}$ of $3 \%$ thioglycollate (SigmaAldrich), and peritoneal lavage was performed after 4 hours as described previously (52). Neutrophil counts were determined by morphological analyses of Diff-Quik-stained (VWR) cytocentrifuge preparations by an observer blind to the genotype.

\section{Statistics}

Results are reported as mean \pm SEM. Statistical significance was assessed by unpaired 2 -tailed Student $t$ test. A $P$ value less than 0.05 was considered significant.

Note added in proof. It was brought to our attention that the loss of CalDAG-GEFI expression in 2 LAD-III patients resulted in aberrant $G$ protein-coupled receptor-triggered $\alpha_{\mathrm{IIb}} \beta_{3}$ activation in platelets and absent $\beta_{2}$ integrin activation in neutrophils ( $R$. Alon and A. Etzioni, unpublished observations).

\section{Acknowledgments}

We are grateful to Tanya Mayadas for advice and helpful discussions and Richard Hynes for critical reading of the manuscript and advice. We thank Lesley Cowan for help with preparing the manuscript. This work was funded by a Scientist Development grant from the American Heart Association (to W. Bergmeier); by stipend GO1360 from Deutsche Forschungsgemeinschaft (to T. Goerge); by National Heart, Lung, and Blood Institute, NIH, grant R37-HL41002 (to D.D. Wagner); by National Institute of Child Health and Develop- 
ment, NIH, grant R01-HD28341 (to A.M. Graybiel); by the Simons Foundation (to A.M. Graybiel); and by National Institutes of Mental Health, NIH, grant F32-MH065815 (to J.R. Crittenden).

Received for publication October 9, 2006, and accepted in revised form February 27, 2007.

1. Hynes, R.O. 2002. Integrins: bidirectional, allosteric signaling machines. Cell. 110:673-687.

2. Sims, T.N., and Dustin, M.L. 2002. The immunological synapse: integrins take the stage. Immunol. Rev. 186:100-117.

3. Simon, S.I., and Green, C.E. 2005. Molecular mechanics and dynamics of leukocyte recruitment during inflammation. Annu. Rev. Biomed. Eng. 7:151-185.

4. Savage, B., Cattaneo, M., and Ruggeri, Z.M. 2001. Mechanisms of platelet aggregation. Curr. Opin. Hematol. 8:270-276.

5. Anderson, D.C., and Springer, T.A. 1987. Leukocyte adhesion deficiency: an inherited defect in the Mac-1, LFA-1, and p150,95 glycoproteins. Annu. Rev. Med. 38:175-194.

6. Tomiyama, Y. 2000. Glanzmann thrombasthenia: integrin alpha IIb beta 3 deficiency. Int. J. Hematol. 72:448-454.

7. Mizgerd,J.P., et al. 1997. Neutrophil emigration in the skin, lungs, and peritoneum: different requirements for CD11/CD18 revealed by CD18-deficient mice. J. Exp. Med. 186:1357-1364.

8. Hodivala-Dilke, K.M., et al. 1999. $\beta 3$-integrin-deficient mice are a model for Glanzmann thrombasthenia showing placental defects and reduced survival. J. Clin. Invest. 103:229-238.

9. Alon, R., and Etzioni, A. 2003. LAD-III, a novel group of leukocyte integrin activation deficiencies. Trends Immunol. 24:561-566.

10. Kuijpers, T.W., et al. 1997. Leukocyte adhesion deficiency type 1 (LAD-1)/variant. A novel immunodeficiency syndrome characterized by dysfunctional $\beta_{2}$ integrins. J. Clin. Invest. 100:1725-1733.

11. Harris, E.S., et al. 2001. A novel syndrome of variant leukocyte adhesion deficiency involving defects in adhesion mediated by beta 1 and beta 2 integrins. Blood. 97:767-776.

12. McDowall, A., et al. 2003. A novel form of integrin dysfunction involving $\beta 1, \beta 2$, and $\beta 3$ integrins. J. Clin. Invest. 111:51-60. doi:10.1172/JCI200314076.

13. Alon, R., et al. 2003. A novel genetic leukocyte adhesion deficiency in subsecond triggering of integrin avidity by endothelial chemokines results in impaired leukocyte arrest on vascular endothelium under shear flow. Blood. 101:4437-4445.

14. Karsan, A., et al. 1998. Leukocyte adhesion deficiency type II is a generalized defect of de novo GDP-fucose biosynthesis. Endothelial cell fucosylation is not required for neutrophil rolling on human nonlymphoid endothelium. J. Clin. Invest. 101:2438-2445.

15. Crittenden, J.R., et al. 2004. CalDAG-GEFI integrates signaling for platelet aggregation and thrombus formation. Nat. Med. 10:982-986.

16. Springett, G.M., Kawasaki, H., and Spriggs, D.R. 2004. Non-kinase second-messenger signaling: new pathways with new promise. Bioessays. 26:730-738.

17. Kawasaki, H., et al. 1998. A Rap guanine nucleotide exchange factor enriched highly in the basal ganglia. Proc. Natl. Acad. Sci. U. S. A. 95:13278-13283.

18. Torti, M., and Lapetina, E.G. 1994. Structure and function of rap proteins in human platelets. Thromb. Haemost. 71:533-543.

19. Quinn, M.T., et al. 1989. Association of a Ras-related protein with cytochrome b of human neutrophils.

Address correspondence to: Denisa D. Wagner or Wolfgang Bergmeier, CBR Institute for Biomedical Research, 800 Huntington Avenue, Boston, Massachusetts 02115, USA. Phone: (617) 278-3344; Fax: (617) 278-3368; E-mail: wagner@cbr.med.harvard.edu (D. Wagner). Phone: (617) 278-6617; Fax: (617) 278-3368; E-mail: bergmeier@cbrinstitute.org (W. Bergmeier).

Nature. 342:198-200.

20. Frenette, P.S., Mayadas, T.N., Rayburn, H., Hynes, R.O., and Wagner, D.D. 1996. Susceptibility to infection and altered hematopoiesis in mice deficient in both P- and E-selectins. Cell. 84:563-574.

21. Knaus, U.G., Heyworth, P.G., Kinsella, B.T., Curnutte, J.T., and Bokoch, G.M. 1992. Purification and characterization of Rac 2. A cytosolic GTPbinding protein that regulates human neutrophil NADPH oxidase. J. Biol. Chem. 267:23575-23582.

22. Abo, A., et al. 1991. Activation of the NADPH oxidase involves the small GTP-binding protein p21rac1. Nature. 353:668-670.

23. Bos, J.L., de Rooij, J., and Reedquist, K.A. 2001. Rap1 signalling: adhering to new models. Nat. Rev. Mol. Cell Biol. 2:369-377.

24. Caron, E. 2003. Cellular functions of the Rap1 GTP-binding protein: a pattern emerges. J. Cell Sci. 116:435-440.

25. Ugarova, T.P., and Yakubenko, V.P. 2001. Recognition of fibrinogen by leukocyte integrins. Ann. N. Y. Acad. Sci. 936:368-385.

26. Butcher, E.C. 1991. Leukocyte-endothelial cell recognition: three (or more) steps to specificity and diversity. Cell. 67:1033-1036.

27. Tager, A.M., et al. 2000. BLTR mediates leukotriene B (4)-induced chemotaxis and adhesion and plays a dominant role in eosinophil accumulation in a murine model of peritonitis. J. Exp. Med. 192:439-446.

28. Coxon, A., et al. 1996. A novel role for the beta 2 integrin CD11b/CD18 in neutrophil apoptosis: a homeostatic mechanism in inflammation. Immunity. 5:653-666.

29. Forlow, S.B., Foley, P.L., and Ley, K. 2002. Severely reduced neutrophil adhesion and impaired host defense against fecal and commensal bacteria in CD18/-P-selectin ${ }^{-/}$double null mice. FASEB J. 16:1488-1496.

30. Walzog, B., Scharffetter-Kochanek, K., and Gaehtgens, P. 1999. Impairment of neutrophil emigration in CD18-null mice. Am. J. Physiol. 276:G1125-G1130.

31. Graf, J.M., Smith, C.W., and Mariscalco, M.M. 1996. Contribution of LFA-1 and Mac-1 to CD18-dependent neutrophil emigration in a neonatal rabbit model. J. Appl. Physiol. 80:1984-1992.

32. Henderson, R.B., et al. 2001. The use of lymphocyte function-associated antigen (LFA)-1-deficient mice to determine the role of LFA-1, Mac-1, and alpha4 integrin in the inflammatory response of neutrophils. J. Exp. Med. 194:219-226.

33. Ruggeri, Z.M. 2002. Platelets in atherothrombosis. Nat. Med. 8:1227-1234.

34. Gruner, S., et al. 2003. Multiple integrin-ligand interactions synergize in shear-resistant platelet adhesion at sites of arterial injury in vivo. Blood. 102:4021-4027.

35. Bergmeier, W., et al. 2002. Flow cytometric detection of activated mouse integrin alphaIIbbeta3 with a novel monoclonal antibody. Cytometry. 48:80-86.

36. Beumer, S., IJsseldijk, M.J., de Groot, P.G., and Sixma, J.J. 1994. Platelet adhesion to fibronectin in flow: dependence on surface concentration and shear rate, role of platelet membrane glycoproteins GP IIb/IIIa and VLA-5, and inhibition by heparin. Blood. 84:3724-3733.

37. Denis, C., et al. 1998. A mouse model of severe von Willebrand disease: defects in hemostasis and thrombosis. Proc. Natl. Acad. Sci. U. S. A. 95:9524-9529.

38. Ni, H., et al. 2000. Persistence of platelet thrombus formation in arterioles of mice lacking both von Willebrand factor and fibrinogen. J. Clin. Invest. 106:385-392

39. Kolanus, W., and Zeitlmann, L. 1998. Regulation of integrin function by inside-out signaling mechanisms. Curr. Top. Microbiol. Immunol. 231:33-49.

40. Quinton, T.M., et al. 2002. Protein kinase C- and calcium-regulated pathways independently synergize with Gi pathways in agonist-induced fibrinogen receptor activation. Biochem. J. 368:535-543.

41. Bos, J.L., et al. 2003. The role of Rap1 in integrin-mediated cell adhesion. Biochem. Soc. Trans. 31:83-86.

42. Geberhiwot, T., et al. 1999. Blood platelets contain and secrete laminin- 8 (alpha4beta1gamma1) and adhere to laminin-8 via alpha6beta1 integrin. Exp. Cell Res. 253:723-732.

43. Bernardi, B., et al. 2006. The small GTPase Rap1b regulates the cross talk between platelet integrin alpha2beta1 and integrin alphaIIbbeta3. Blood. 107:2728-2735.

44. Nieswandt, B., Bergmeier, W., Rackebrandt, K., Gessner, J.E., and Zirngibl, H. 2000. Identification of critical antigen-specific mechanisms in the development of immune thrombocytopenic purpura in mice. Blood. 96:2520-2527.

45. Ware, J., Russell, S., and Ruggeri, Z.M. 2000. Generation and rescue of a murine model of platelet dysfunction: the Bernard-Soulier syndrome. Proc. Natl. Acad. Sci. U. S. A. 97:2803-2808.

46. Kinashi, T., et al. 2004. LAD-III, a leukocyte adhesion deficiency syndrome associated with defective Rap1 activation and impaired stabilization of integrin bonds. Blood. 103:1033-1036.

47. Chrzanowska-Wodnicka, M., Smyth, S.S., Schoenwaelder, S.M., Fischer, T.H., and White, G.C., 2nd. 2005. Rap1b is required for normal platelet function and hemostasis in mice [erratum 2005, 115:2296]. J. Clin. Invest. 115:680-687. doi:10.1172/ JCI200522973.

48. Liu, S., Calderwood, D.A., and Ginsberg, M.H. 2000. Integrin cytoplasmic domain-binding proteins. J. Cell Sci. 113:3563-3571.

49. Ratnikov, B.I., Partridge, A.W., and Ginsberg, M.H. 2005. Integrin activation by talin. J. Thromb. Haemost. 3:1783-1790.

50. Wu, C., and Dedhar, S. 2001. Integrin-linked kinase (ILK) and its interactors: a new paradigm for the coupling of extracellular matrix to actin cytoskeleton and signaling complexes. J. Cell Biol. 155:505-510.

51. Bradley, P.P., Priebat, D.A., Christensen, R.D., and Rothstein, G. 1982. Measurement of cutaneous inflammation: estimation of neutrophil content with an enzyme marker. J. Invest. Dermatol. 78:206-209.

52. Mayadas, T.N., Johnson, R.C., Rayburn, H., Hynes, R.O., and Wagner, D.D. 1993. Leukocyte rolling and extravasation are severely compromised in $\mathrm{P}$ selectin-deficient mice. Cell. 74:541-554. 